\title{
Injective chromatic number of lexicographic product of two graphs
}

\author{
Bhanupriya $\mathrm{C} \mathrm{K}^{1 *}$ and Sunitha $\mathrm{M} \mathrm{S}^{2}$
}

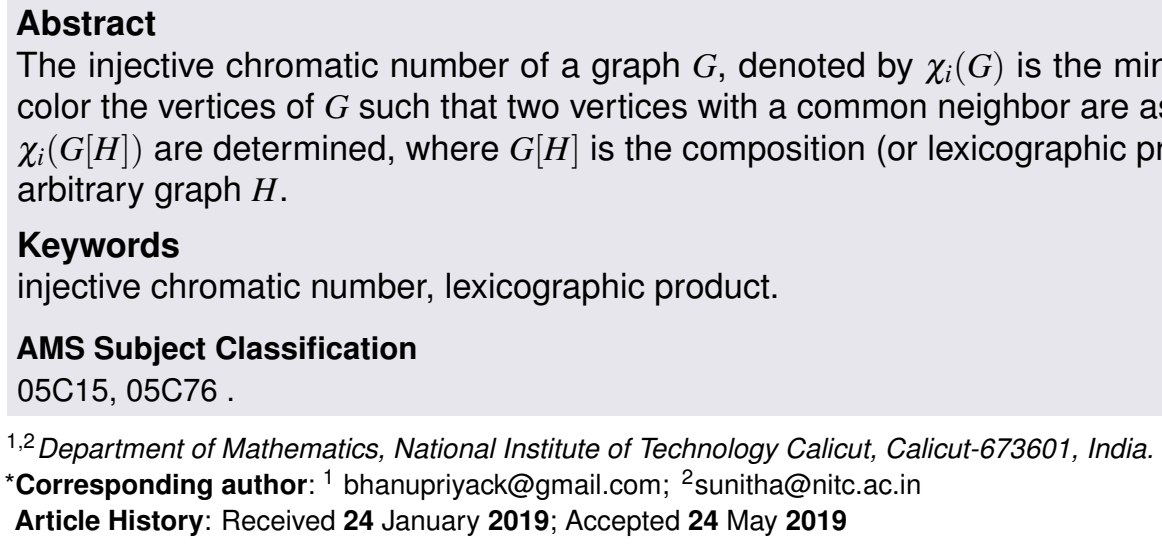
arbitrary graph $H$.

\section{Keywords}

injective chromatic number, lexicographic product.

\section{AMS Subject Classification} 05C15, $05 \mathrm{C} 76$.

1,2 Department of Mathematics, National Institute of Technology Calicut, Calicut-673601, India.

*Corresponding author: ${ }^{1}$ bhanupriyack@gmail.com; ${ }^{2}$ sunitha@nitc.ac.in

Article History: Received 24 January 2019; Accepted 24 May 2019 color the vertices of $G$ such that two vertices with a common neighbor are assigned distinct colors. In this paper $\chi_{i}(G[H])$ are determined, where $G[H]$ is the composition (or lexicographic product) of $G\left(P_{n}, C_{n}\right.$ and $\left.K_{n}\right)$ with any

\section{Contents}

1

3

Introduction 476

Preliminaries

476

References 476 478

\section{Introduction}

The basic definitions of graph theory are taken from [2]. The concepts of injective coloring and injective chromatic number $\chi_{i}(G)$ of a graph $G$ are introduced by G.Hahn et.al [1]. The authors established some upper and lower bounds for injective chromatic number $\chi_{i}(G)$ and are obtained the injective chromatic number of the hypercubes also. In [5] B.Luzar et.al obtained some results on injective coloring of planar graphs with large girth and few colors. Seog-Jin kim et.al [3] proved that injective chromatic number of $G$ is at least half of the chromatic number of $G^{2}$, the square of $G$. The injective chromatic sum $\sum_{i}(G)$ and injective strength $s_{i}(G)$ are introduced by A. Kishore and M.S. Sunitha [4]. The authors obtained the injective chromatic sum of some classes of graphs, suggested bounds for injective chromatic sum and established injective chromatic sum of graph complements, join, union, product and corona. The concept of injective chromatic polynomial is also introduced in this paper. J. Song and J. Yue. [6] obtained some sharp bounds (or exact values) of injective chromatic number of Cartesian product, direct product, lexicographic product, union, join, and disjunction of graphs.

In this paper $\chi_{i}(G[H])$ are determined, where $G[H]$ is the composition (or lexicographic product) of $G\left(P_{n}, C_{n}\right.$ and $\left.K_{n}\right)$ with any arbitrary graph $H$.

\section{Preliminaries}

The concepts of injective coloring and injective chromatic number are introduced in 2002 by G. Hahn et.al and it is defined as follows:

Definition 2.1. [1] An injective coloring of a graph $G=$ $(V, E)$ is a coloring of the vertices of $G$ that assigns different colors to any pair of vertices that have a common neighbor.

Definition 2.2. [1] The injective chromatic number $\chi_{i}(G)$ of a graph $G=(V, E)$ is the minimum number of colors needed to color the vertices of $G$ such that two vertices with a common neighbor are assigned distinct colors.

Definition 2.3. [2] The composition (or lexicographic product) $G=G_{1}\left[G_{2}\right]$ of two graphs $G_{1}=\left(V_{1}, E_{1}\right)$ and $G_{2}=\left(V_{2}, E_{2}\right)$ has $V=V_{1} \times V_{2}$ as its vertex set, and $u=\left(u_{1}, u_{2}\right)$ is adjacent with $v=\left(v_{1}, v_{2}\right)$ whenever $u_{1}$ adjacent $v_{1}$ or $u_{1}=v_{1}$ and $u_{2}$ adjacent $v_{2}$. 


\section{Main Results}

In this section the injective chromatic number of lexicographic products are obtained. For any two arbitrary graphs $G$ and $H$, the injective chromatic number of $G[H]$ is bounded, it is established in [6].

Remark 3.1. [6] Let $G$ and $H$ be graphs. Then $\chi_{i}(G[H]) \leq(\Delta+1) \chi_{i}(H)$ and the bound is sharp.

The following theorem shows that the injective chromatic number of $K_{n}[G]$ is the total number of vertices of $K_{n}[G]$.

Theorem 3.2. For any connected graph $G$ with at least 2 vertices,

$$
\chi_{i}\left(K_{n}[G]\right)=n|V(G)|, n \geq 2 .
$$

Proof. Let $v_{1}, v_{2}, \ldots, v_{n}$ be the vertices of $K_{n}$ and $u_{1}, u_{2}, \ldots, u_{m}$ be the vertices of $G$. The vertex set of $K_{n}[G]$ is

$V=\left\{\left(v_{1}, u_{1}\right),\left(v_{1}, u_{2}\right), \ldots,\left(v_{1}, u_{m}\right)\right\} \cup\left\{\left(v_{2}, u_{1}\right),\left(v_{2}, u_{2}\right), \ldots\right.$, $\left.\left(v_{2}, u_{m}\right)\right\} \cup \ldots \cup\left\{\left(v_{n}, u_{1}\right),\left(v_{n}, u_{2}\right), \ldots,\left(v_{n}, u_{m}\right)\right\}=V_{1} \cup V_{2} \cup$ $\ldots \cup V_{n}$. Note that any vertex $\left(v_{j}, u_{k}\right), 1 \leq k \leq m$ is a common neighbor of any two vertices in $V_{i}, 1 \leq i, j \leq n, i \neq j$ and thus $m$ distinct colors are needed to color the vertices in $V_{i}$. Since $G$ is a connected graph, $N\left(u_{k}\right) \neq \phi, 1 \leq k \leq m$ and let $u_{l} \in N\left(u_{k}\right)$. Then there is a path $\left(v_{i}, u_{k}\right)\left(v_{i}, u_{l}\right)\left(v_{j}, u_{s}\right)$, $1 \leq s \leq m$ of length 2 . Thus the vertices in any two sets $V_{i}$ and $V_{j}, 1 \leq i, j \leq n, i \neq j$ cannot have vertices with same colors. Thus each set $V_{i}, 1 \leq i \leq n$ need distinct $m$ colors and hence $\chi_{i}\left(K_{n}[G]\right)=n m=n|V(G)|$.

The injective chromatic number of lexicographic product of $P_{n}$ with any arbitrary connected graph $G$ is obtained as follows.

Theorem 3.3. For any connected graph $G$ with at least 2 vertices,

$$
\chi_{i}\left(P_{n}[G]\right)= \begin{cases}2|V(G)| & \text { for } n=2 \\ 3|V(G)| & \text { otherwise. }\end{cases}
$$

Proof. Let $v_{1}, v_{2}, . ., v_{n}$ be the vertices of $P_{n}$ and $u_{1}, u_{2}, . ., u_{m}$ be the vertices of $G$. The vertex set of $P_{n}[G]$ is

$V=\left\{\left(v_{1}, u_{1}\right),\left(v_{1}, u_{2}\right), \ldots,\left(v_{1}, u_{m}\right)\right\} \cup\left\{\left(v_{2}, u_{1}\right),\left(v_{2}, u_{2}\right), \ldots\right.$, $\left.\left(v_{2}, u_{m}\right)\right\} \cup \ldots \cup\left\{\left(v_{n}, u_{1}\right),\left(v_{n}, u_{2}\right), \ldots,\left(v_{n}, u_{m}\right)\right\}=V_{1} \cup V_{2} \cup \ldots \cup$ $V_{n}$. Any vertex in $V_{2}$ is a common neighbor for the vertices in $V_{1}$, then $m$ distinct colors are needed to color the vertices in $V_{1}$. Let $i$ be the color of the vertices $\left(v_{1}, u_{i}\right), 1 \leq i \leq m$. Now consider the vertices in $V_{2}$. Since $G$ is a connected graph, $N\left(u_{i}\right) \neq \phi$ and let $u_{j} \in N\left(u_{i}\right)$, then $\left(v_{2}, u_{i}\right)\left(v_{2}, u_{j}\right)\left(v_{1}, u_{k}\right)$, $1 \leq i, k \leq m$ form a path of length 2 . Thus any color of $\left(v_{1}, u_{j}\right)$ cannot be the color of vertices in $V_{2}$. Thus a new set of $m$ colors are needed to color the vertices in $V_{2}$. Let $m+i$ be the color of $\left(v_{2}, u_{i}\right) 1 \leq i \leq m$. Now consider the vertices in $V_{3}$. Here $\left(v_{3}, u_{i}\right)\left(v_{3}, u_{j}\right)\left(v_{2}, u_{k}\right), 1 \leq i, k \leq m$ form a path of length 2 . Thus any color of vertices in $V_{2}$ cannot be the color of the vertices in $V_{3}$. Also $\left(v_{3}, u_{i}\right)\left(v_{3}, u_{j}\right)\left(v_{1}, u_{k}\right), 1 \leq i, j, k \leq m$ form a path of length 2, the vertices in $V_{3}$ cannot be colored with any of the color of the vertices in $V_{1}$. Now use the colors of the vertices in $V_{1}, V_{2}$ and $V_{3}$ for the vertices in $V_{3 i+1}, V_{3 i+2}$ and $V_{3(i+1)}$ respectively. Hence a total of $3 m$ distinct colors are needed for an injective coloring of $P_{n}[G]$.

The lexicographic product $C_{n}[G]$ of a cycle and a connected graph has invective chromatic number as follows.

Theorem 3.4. For any connected graph $G$ with at least 2 vertices,

$$
\chi_{i}\left(C_{n}[G]\right)= \begin{cases}3|V(G)| & \text { for } n \equiv 0(\bmod 3) \\ 4|V(G)| & \text { for } n \equiv 1(\bmod 3) \\ 5|V(G)| & \text { for } n \equiv 2(\bmod 3) .\end{cases}
$$

Proof. Let $v_{1}, v_{2}, \ldots, v_{n}$ be the vertices of $C_{n}$ and $u_{1}, u_{2}, \ldots, u_{m}$ be the vertices of $G$. The vertex set of $C_{n}[G]$ is $V=\left\{\left(v_{1}, u_{1}\right),\left(v_{1}, u_{2}\right),\left(v_{1}, u_{3}\right), \ldots,\left(v_{1}, u_{m}\right)\right\} \cup\left\{\left(v_{2}, u_{1}\right)\right.$, $\left.\left(v_{2}, u_{2}\right),\left(v_{2}, u_{3}\right), \ldots,\left(v_{2}, u_{m}\right)\right\} \cup \ldots \cup\left\{\left(v_{n}, u_{1}\right),\left(v_{n}, u_{2}\right),\left(v_{n}, u_{3}\right)\right.$, $\left.\ldots,\left(v_{n}, u_{m}\right)\right\}=V_{1} \cup V_{2} \cup V_{3} \cup \ldots \cup V_{n}$.

Case i: $n \equiv 0(\bmod 3)$

Any vertex in $V_{i}$ is a common neighbor for the vertices in $V_{i-1}$ and $V_{i+1}$. Thus $2 m$ distinct colors are needed to color the vertices in $V_{i-1}$ and $V_{i+1}$. Since $G$ is connected, $N\left(u_{k}\right) \neq \phi$ and let $u_{j} \in N\left(u_{k}\right)$. Then $\left(v_{i}, u_{k}\right)\left(v_{i}, u_{j}\right)\left(v_{i+1}, u_{l}\right)$ and $\left(v_{i}, u_{k}\right)\left(v_{i}, u_{j}\right)$ $\left(v_{i-1}, u_{l}\right)$ form paths of length 2 for $1 \leq l \leq m$. Thus $\left(v_{i}, u_{k}\right)$, $1 \leq k \leq m$ cannot be colored with the color used for $\left(v_{i+1}, u_{l}\right)$ and $\left(v_{i-1}, u_{l}\right), 1 \leq l \leq m$. Thus a new set of $m$ colors are needed to color the vertices $\left(v_{i}, u_{k}\right), 1 \leq k \leq m$. Hence a total of $3 m$ colors are used to color the vertices of $C_{n}[G]$ and thus $\chi_{i}\left(C_{n}[G]\right)=3 m=3|V(G)|$. Thus the injective coloring of $C_{n}[G]$ is given as follows, for $i=3 k+1$ and $0 \leq k<\frac{\left|C_{n}[G]\right|}{3}$ and $1 \leq j \leq m$

- color $j$ is given to the vertices $\left(v_{i}, u_{j}\right)$

- color $m+j$ is given to the vertices $\left(v_{i+1}, u_{j}\right)$

- color $2 m+j$ is given to the vertices $\left(v_{i+2}, u_{j}\right)$

Case ii: $n \equiv 1(\bmod 3)$ i.e. $n=3 k+1$

Here the vertex set of $C_{n}[G]$ is $V=V_{1} \cup V_{2} \cup \ldots \cup V_{3 k} \cup V_{3 k+1}$. The vertices in $V_{1}, V_{2}, \ldots, V_{3 k}$ can be colored as in Case i. Now the uncolored vertices are the vertices in $V_{3 k+1}$, which cannot be colored with any of the colors that are used for the vertices in $V_{1}, V_{2} V_{3 k}$ and $V_{3 k-1}$, since these vertices have common neighbor with the vertices in $V_{3 k+1}$. Thus a new set of $m$ colors are needed to color these vertices. Hence a total of $4 m$ colors are needed for the injective coloring.

Case iii: $n \equiv 2(\bmod 3)$ i.e. $n=3 k+2$

Here the vertex set of $C_{n}[G]$ is $V=V_{1} \cup V_{2} \cup \ldots \cup V_{3 k+1} \cup V_{3 k+2}$. Color the vertices in $V_{1}, V_{2}, \ldots V_{3 k+1}$ as in case ii. Now the uncolored vertices are the vertices in $V_{3 k+2}$, which cannot be colored with any of the colors that are used for the vertices in $V_{1}, V_{2}, V_{3 k}$, and $V_{3 k+1}$. Since these set of vertices have common neighbor with the vertices in $V_{3 k+2}$. Thus color the vertices in $V_{3 k+2}$ with a new set of $m$ colors. Hence a 
total of $5 \mathrm{~m}$ colors are needed for an injective coloring of $C_{3 k+2}[G]$.

\section{Conclusion}

In this paper $\chi_{i}(G[H])$ are determined, where $G[H]$ is the composition (or lexicographic product) of two graphs $G$ and $H$. Where $G$ is $P_{n}, C_{n}$ or $K_{n}$ and $H$ is any arbitrary graph.

\section{References}

[1] Gena Hahn, Jan Kratochvil, Jozef Siran and Dominique Sotteau, On the injective chromatic number of graphs, Discrete Mathematics, 256(1-2)(2002), 179-192.

[2] Frank Harary, Graph Theory. Adison-Wesley Puv. Co. Inc., Philippines, 1969.

[3] Seog-Jin Kim and Sang-il Oum, Injective chromatic number and chromatic number of the square of graphs, Preprint, 2009.

[4] Anjaly Kishore and M S Sunitha, Injective chromatic sum and injective chromatic polynomials of graphs, General Mathematics Notes, 18(2) (2013), 55-66.

[5] Borut Luzar, Riste Skrekovski and Martin Tancer, Injective colorings of planar graphs with few colors, Discrete Mathematics, 309(18)(2009), 5636-5649.

[6] Jiamei Song and Jun Yue, Injective coloring of some graph operations, Applied Mathematics and Computation, 264(2015), 279-283.

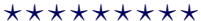

$\operatorname{ISSN}(\mathrm{P}): 2319-3786$

Malaya Journal of Matematik

$\operatorname{ISSN}(\mathrm{O}): 2321-5666$

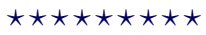

